\title{
Die deutsche Version der Bath Body Perception Disturbance Scale (BBPDS-D)
}

\section{Übersetzung, kulturelle Anpassung und linguistische Validierung an Patienten mit komplexem regionalem Schmerzsyndrom}

M. Tschopp ${ }^{1}$, J. Swanenburg ${ }^{2}$, M.W. Wertli ${ }^{3,4}$, A. Langenfeld ${ }^{2,5}$, C.M. McCabe Candy ${ }^{6,7}$, J. Lewis Jenny $^{6,7}$, E. Baertschi ${ }^{8}$, F. Brunner ${ }^{1}$

' Universitäre Klinik für Rheumatologie, Abteilung für Physikalische Medizin und Rheumatologie, Universitätsklinik Balgrist, Zürich

${ }^{2}$ Interdisziplinäre Wirbelsäulenforschung, Abteilung für Chiropraktische Medizin, Universität Zürich und Universitätsklinik Balgrist, Zürich

${ }^{3}$ Horten Zentrum für Wissenstransfer, Universität Zürich, Zürich

${ }^{4}$ Abteilung für Allgemeine Innere Medizin, Universitätsspital Bern, Universität Bern

${ }^{5}$ Abteilung für Epidemiologie und CAPHRI (School for Public Health and Primary Care),

Universität Maastricht

${ }^{6}$ Royal United Hospitals NHS Foundation Trust, Bath

${ }^{7}$ Faculty of Health and Life Sciences, University of West of the England, Bristol

${ }^{8}$ Abteilung für Physiotherapie, Universitätsklinik Balgrist, Zürich

\section{Korrespondenzadresse:}

Dr. med. Marcel Tschopp

Universitäre Klinik für Rheumatologie

Abteilung für Physikalische Medizin und Rheumatologie 
Universitätsklinik Balgrist

Forchstrasse 340

CH-8008 Zürich

Schweiz

Telefon: +41443863508

Marcel.Tschopp@Balgrist.ch

[Abbildung 1]

\section{Danksagung}

Die Autoren danken der Abteilung Physiotherapie der Universitätsklinik Balgrist für ihre Unterstützung bei der Rekrutierung der Probanden.

\section{Interessenkonflikt}

Der korrespondierende Autor gibt an, dass kein Interessenkonflikt besteht. 


\section{Zusammenfassung}

Hintergrund: Störungen der Körperwahrnehmung stellen häufige Begleitsymptome bei Patienten mit komplexem regionalem Schmerzsyndrom (CRPS) dar. Mit der Bath Body Perception Disturbance Scale (BBPDS) steht ein nützliches Instrument zur Erfassung von Körperwahrnehmungstörungen zur Verfügung.

Fragestellung: Das Ziel dieser Studie besteht darin, die Originalversion der BBPDS aus dem Englischen in die deutsche Sprache zu übersetzen, kulturell anzupassen und eine linguistische Validierung bei Patienten mit akutem CRPS (Symptome $<3$ Monate) oder stabilem CRPS (Symptome $\geq 3$ Monate) durchzuführen.

Material und Methoden: Die englische Originalversion wurde gemäss etablierten Richtlinien (Übersetzung und Rückübersetzung) ins Deutsche übersetzt und an 56 Patienten (Durchschnittsalter 50,9 $\pm 13,1$ Jahre) mit akutem $(\mathrm{N}=28)$ oder stabilem CRPS $(\mathrm{N}=28)$ getestet.

Ergebnisse: Die relative Reliabilität, Intra-Klassen-Korrelation und Test-RetestZuverlässigkeit erwies insgesamt und in der Gruppe mit akutem bzw. stabilem CRPS als ausgezeichnet . Die kleinste nachweisbare Änderung lag bei 10,0 Punkten. Beim Test-Retest lagen 48 Punkte innerhalb des 95-Prozent-Konfidenzintervalls und die Sichtprüfung zeigte keine Tendenz zur Heteroskedastizität. Der Spearman- $\rho$-Koeffizient wies keine Korrelation zwischen dem Gesamtwert der deutschen Übersetzung $(\rho=-0,19)$ und dem EuroQol-5 D ( $\rho=$ 0,16) auf. Es gab keine signifikanten Unterschiede zwischen Patienten mit akutem und stabilem CRPS $(\rho=0.412)$. Zudem gab es auch keine Boden- oder Deckeneffekte. 
Schlussfolgerung: Die vorliegende deutsche Übersetzung und interkulturelle Anpassung der BBPDS stellt ein valides Instrument dar, um Körperwahrnehmungsstörungen bei deutschsprachigen CRPS Patienten zu erfassen. In zukünftigen Forschungsprojekten sollte der Einfluss von Körperwahrnehmungsstörungen auf das Behandlungsergebnis und die Prognose des CRPS weiter untersucht werden. 


\section{Schlüsselwörter}

Komplexes regionales Schmerzsyndrom

Körperwahrnehmungsstörung

Bath Body Perception Disturbance Scale

Bath Körperwahrehmungsstörung Skala 


\begin{abstract}
Background: Besides the classical clinical manifestations, body perception disturbances are common among patients with Complex Regional Pain Syndrome (CRPS). The Bath Body Percetption Disturbance Scale represents a useful tool to assess these changes in CRPS patients. However, to date no valdiated German version is available.
\end{abstract}

Objective: The aim of this study was to translate the Bath Body Perception Disturbance Scale into German, to perform a cross-cultural adaptation, and linguistic validation in acute (symptoms $<3$ months) and stable CRPS (symptoms $\geq 3$ months).

Materials and methods: We translated the Bath Body Perception Disturbance Scale according to published guidelines.

Results: Fifty-six patients (49 females, mean age $50.9 \pm 13.1$ years) with acute $(\mathrm{N}=28)$ and stable CRPS (N=28) were included in this study. Relative reliability, internal consistency and test-retest reliability was excellent overall, in acute CRPS and stable CRPS patients. Overall smallest detectable change was 10.0, respectively 10.7 in acute and 9.9 in stable Complex Regional Pain Syndrome 1 patients. Forty-eight points lay within the 95\% limits of agreement for test-retest and visual inspection showed no tendency towards heteroscedasticity. Spearman's $\rho$ coefficient values showed no correlation between the total score of the German translation $(\rho=-0.19)$ and the EuroQol-5 D $(\rho=0.16)$. There were no significant differences between patients with acute and stable CRPS $(\rho=0.412)$. There were no floor or ceiling effects.

Conclusion: This German translation and cross-cultural adaptation of the original English version of the Bath Body Perception Disturbance Scale is a valid instrument to assess body perception disturbances in German speaking CRPS patients. Future research should further assess impact of body perception disturbance on treatment outcome and prognosis. 


\section{Keywords}

Complex regional pain syndrome

Body perception disturbances

Bath Body Perception Disturbance Scale 


\section{Hintergrund und Fragestellung}

Der Begriff des komplexen regionalen Schmerzsyndromes (CRPS) umschreibt verschiedene schmerzhafte Zustände, die typischerweise nach einem auslösenden Ereignis an Extremitäten auftreten können [1]. Charakteristischerweise übersteigt dabei die Dauer und Intensität der Beschwerden den normalerweise zu erwartenden Verlauf [1]. Die Erkrankung kann für die Betroffenen mit einer wesentlicher Funktionseinschränkung im Alltag und einer eingeschränkten Lebensqualität verbunden sein. Beim CRPS werden zwei Typen voneinander unterschieden: Während beim CRPS 1 keine Nervenläsion nachweisbar ist, liegt beim CRPS 2 eine wesentliche Verletzung eines Nerven oder eines Nervenhauptstammes vor. Aus pathophysiologischer Sicht werden als zugrunde liegenden Mechanismen eine übermässige neurogene Entzündungsreaktion, eine vasomotorische Dysfunktion und eine kortikale Reorganisation vermutet, wobei sich diese Prozesse gegenseitig beeinflussen [2]. Klinisch manifestiert sich das CRPS mit einem bunten Symptomkomplex in Form von sensiblen, vaso- bzw. sudomotorischen, motorischen und trophischen Veränderungen [3]. Neben diesen Manifestationen entwickeln 54,4\% bis $84 \%$ der CRPS Patienten eine veränderte Wahrnehmung gegenüber der betroffenen Extremität [4-6]. Solche Wahrnehmungsstörungen, welche im Englischen als ,body perception disturbances' bezeichnet werden, äussern sich in Form eines veränderten Bewusstseins der tangierten Gliedmasse in Bezug auf Grösse, Form, Gewicht und Temperatur. Daneben entwickeln die Betroffenen oft eine Abneigung bzw. Fremdkörpergefühle gegenüber der betroffenen Extremität [7], welche mit einem Wunsch nach Amputation einhergehen kann [4, 5, 7, 8]. Diese Symptome können sich lediglich unterschwellig auftreten und sind häufig nur durch ein direktes Ansprechen in der Anamnese erfassbar [6].

Im klinischen Alltag stellt sich deshalb die Auseinandersetzung von CRPS Patienten mit ihrer betroffenen Extremität vielfach als Herausforderung dar, was sich potentiell negativ auf das 
Behandlungsergebnis auswirken kann. Lewis et al. stellte 2012 in ihrer Untersuchung fest, dass CRPS Patienten mit Körperwahrnehmungsstörungen an einer höheren Schmerzintensität und eine längere Krankheitsdauer aufwiesen als Patienten ohne Körperwahrnehmungsstörungen [9].

Die Bath Körperwahrnehmungsstörungs Skala (englisch: Bath Body Perception Disturbance Skala) (BBPDS) stellt ein nützliches Instrument, um bei Patienten mit CRPS eine veränderte Körperwahrnehmungsstörungen zu erfassen und zu quantifizieren [10]. Das Ziel dieser Studie bestand darin die englische Originalversion der BBPDS in die deutsche Sprache zu übersetzen, eine kulturelle Anpassung vorzunehmen und die Validität der deutschen Version zu überprüfen. 


\section{Patienten und Methoden}

\section{Teilnehmer}

Die Probanden dieser Studie wurden zwischen 2013 und 2016 fortlaufend aus der ambulanten Sprechstunde der Abteilung für Physikalische Medizin und Rheumatologie der Universitätsklinik Balgrist rekrutiert. Zur Qualitätskontrolle wurde eine Gruppe von gesunden, deutschsprachigen Teilnehmern aus dem medizinischen und therapeutischen Bereich der Universitätsklinik Balgrist eingeschlossen. Die Diagnose des CRPS erfolgte nach den aktuell gültigen Diagnosekriterien (überarbeitete Budapest Kriterien) [11]. Basierend auf der Symptomdauer wurde zwischen Patienten mit akutem CRPS (Symptome $<3$ Monate) und stabilem CRPS (Symptome $\geq 3$ Monate) unterschieden. Eingeschlossen wurden alle potentiell geeigneten Patienten mit CRPS, welche bisher nicht mit der BBPDS vertraut waren und älter als 18 Jahre waren. Als Ausschlusskriterium galt das nicht Beherrschen der Deutschen Sprache und Patienten mit schweren psychischen Störungen.

Alle Probanden gaben eingangs ihre schriftliche Zustimmung zur Teilnahme an dieser Studie. Diese Studie wurde von der Ethikkommission des Kantons Zürich genehmigt (KEK-ZH-Nr: 2013-0002).

\section{Die Bath Body Perception Disturbance Scale (BBPDS)}

Die BBPDS wurde zur Erfassung und Quantifizierung von Körperwahrnehmungsstörungen bei CRPS Patienten entwickelt [9]. Die englische Originalversion ist im Anhang 1 abgebildet.

Die Fragen 1 bis 4 und 6b quantifizieren Störungen auf einer Skala von 0 bis $10(0=$ Minimum, 10 = Maximum). Die Fragen 5 und 6 werden mit Ja (1 Punkt) oder Nein (0 Punkte) beantwortet. Zur Beantwortung von Frage 7 wird der Patient gebeten, die betroffene Extremität mit geschlossenen Augen bildlich zu beschreiben. Auf der Basis dieser Beschreibung fertigt der Untersucher eine Zeichnung an, welche dann vom Patienten auf 
deren Korrektheit geprüft und schliesslich gutgeheissen wird. Die Zeichnung wird anschliessend vom Untersucher auf einer 3-Punkte-Skala ausgewertet (Keine Verzerrung $=0$, mässige Verzerrung =1, schwere Verzerrung =2). Die maximal erreichbare Punktzahl beträgt 57 Punkte. Je höher die erreichte Punktzahl, desto ausgeprägter besteht eine Körperwahrnehmungsstörungen (Anhang 1 und 2).

\section{Übersetzungsprozess}

Zunächst wurde bei den Urheberinnen die Erlaubnis zur Übersetzung BBPDS eingeholt [9] und zur Mitarbeit eingeladen.

Die Übersetzung und die interkulturelle Anpassung folgten etablierten Richtlinien [12]. Dabei verwendeten wir den Ansatz in Form einer sequentiellen Vorwärts- und Rückwärtsübersetzung. Zwei unabhängige, professionelle Übersetzer übersetzten die englische Originalversion der BPDS in die deutsche Sprache. Darauf wurde diese erste Übersetzung im Rahmen eines Konsenstreffens innerhalb eines Expertenteams bezüglich Konsistenz und Validität bewertet. Das Expertenteam setzte sich aus einem betroffenen CRPS Patienten, einer Physiotherapeutin, einem klinischen Forscher und einem Facharzt für Physikalische Medizin und Rehabilitation zusammen. Die Teilnehmer einigten sich auf eine erste deutsche Version (BBPDS-G). Um allfällige Verständnis- oder Interpretationsschwierigkeiten zu identifizieren, wurde die Skala an drei CRPS Patienten geprüft. In einem nächsten Schritt übersetzte ein bisher nicht involvierter, zweisprachiger Mitarbeiter mit biomedizinischem Hintergrund die Skala zurück ins Englische. Diese RückÜbersetzung wurde vom gleichen Expertenteam auf konzeptionelle Unstimmigkeiten geprüft und nach erfolgtem Konsensus an weiteren drei CRPS Patienten und fünfzehn gesunden Probanden getestet. Nach erneuter Analyse der Kommentare und weiteren Anpassungen 
einigte sich das Expertenteam schliesslich auf die vorliegende, definitive deutsche Version der BBPDS-D (siehe Anhang 2).

\section{Validitäsprüfung}

Anlässlich einer Konsultation in der Ambulanz wurden die demographischen Merkmale der Teilnehmer erhoben und den Probanden mündlich präzise Instruktionen zur Beantwortung des BBPDS-D erteilt. Zudem wurden die Teilnehmer im Rahmen der Konsultation gebeten, ihre aktuelle Schmerzintensität auf der numerischen Ratingskala (NRS, numeric rating scale) $(0=$ kein Schmerz, $100=$ maximaler Schmerz) anzugeben und zur Erfassung des aktuellen Gesundheitszustandes den EuroQol-5D-Fragebogen auszufüllen [13]. Alle Teilnehmer wurden angewiesen, die Skala nach drei Tagen ein zweites Mal auszufüllen und dann per Post zurückzusenden. Diejenigen Teilnehmer, die nicht innerhalb von sieben Tagen geantwortet haben, wurden nicht in die Analyse eingeschlossen.

\section{Datenanalyse und Statistik}

Die Charakteristika der Teilnehmer wurden mittels deskriptiver Statistik beschrieben. Die relative Zuverlässigkeit erfolgte mittels Berechnung der Intra-Klassen-Korrelation $\left(\mathrm{ICC}_{2,1}\right.$, intraclass correlation) mit seinem 95-Prozent Konfidenzintervall (CI, confidence interval) [14]. $\mathrm{ICC}_{2,1}$-Ergebnisse $>0,75$ werden als ausgezeichnet interpretiert [15]. Die interne Konsistenz und Zuverlässigkeit wurde durch die Berechnung der Crohnbachs-Alpha-Werte und mit Hilfe der Resultate aus dem ersten Datensatz der BBPDS-D ermittelt. Die Crohnbach Alpha-Werte wurden folgendermassen interpretiert: Exzellent $(>0,9)$, gut $(>0,8)$, akzeptabel $(>0,7)$, fragwürdig $(>0,6)$, schlecht $(>0,5)$ oder inakzeptabel $(<0,5)[16]$.

Zur Beurteilung der absoluten Zuverlässigkeit wurden die minimale messbare Veränderung (SDC, smallest detectable changes) berechnet. Die SDC stellt die minimale Veränderung dar, 
welche der Patient bei den Resultaten der Skala zu verschiedenen Zeitpunkten erreichen muss. Dadurch wird sichergestellt, dass die Änderung real ist und nicht aus einer Messungenauigkeit resultiert [16]. Die SDC wird mit Hilfe der Formel 1,96 x $\sqrt{2} \times$ SEM (standard error of the mean, Standardfehler) kalkuliert. Der SEM wiederum wird aus der gepoolten Standardabweichung (SD, standard deviation) der ersten und zweiten Beurteilung $\sqrt{ }$ (1-ICC) berechnet. Die Test-Retest-Zuverlässigkeit wurde durch visuelle Interpretation des Bland-Altman Plot beurteilt [17]. Die Gültigkeit wurde durch Korrelation der BPDS-D mit den Resultaten des EuroQol-5D geprüft. Der Spearmans-Koeffizient wird als hervorragend $(>0,9)$, gut $(0,7-0,9)$, mittel $(0,5-0,69)$, schwach $(0,2-0,5)$ oder minimal bis nicht mehr vorhanden $(0,0-0,2)$ interpretiert [18]. Für die Beurteilung der Konstruktvalidität und Unterscheidungen zwischen akutem bzw. stabilem CRPS wurde der Mann-Whitney-U-Test verwendet. Boden- oder Deckeneffekte wurden als nachgewiesen erachtet, wenn mehr als 15 Prozent der Ergebnisse die maximal oder minimal mögliche Gesamtpunktzahl erreichten [14]. Alle Analysen wurden mit Hilfe der statistischen Software IBM SPSS 19 (SPSS Inc., Chicago, IL) durchgeführt. Das Signifikanzniveau-Alpha wurde auf 0,05 gesetzt. 


\section{Ergebnisse}

\section{Teilnehmer}

Achtundfünfzig Patienten mit CRPS 1 wurden für die Studie rekrutiert. Zwei Teilnehmer wurden ausgeschlossen, da sie den Fragebogen trotz erneuter Kontaktaufnahme nicht zurückgesendet hatten. Insgesamt wurden 56 Patienten in die Analyse einbezogen (49 Frauen, 7 Männer, Durchschnittalter 50,9 \pm 13,1 Jahre, Alterspanne 18 bis 77 Jahre). Alle Patienten litten an einem CRPS 1. Die Probanden haben jeweils alle Fragen des BPDS-D beantwortet, so dass keine fehlenden Daten vorlagen. Die demographischen und klinischen Merkmale der Teilnehmer sind in Tabelle 1 zusammengefasst.

\section{Übersetzungsprozess und kulturelle Adapation}

Die endgültige Version der BBPDS-D wurde an drei CRPS Patienten und 15 gesunden Probanden vorgeprüft. Basierend auf den Rückmeldungen der Patienten wurden vier kleinere Anpassungen vorgenommen (,Wie ausgeprägt“ zu „,wie stark“, „Extremität“ zu „Gliedmasse“, „Beachtung“ zu „Aufmerksamkeit“, „Gefühle“ zu „Emotionen“). Die gesunden Teilnehmer waren sich einig, dass die Fragen einfach zu verstehen und die Anweisungen ausreichend waren. Einzig die Übersetzung der Frage 5, welche sich auf den Wunsch bezieht, eine Extremität amputieren zu lassen, war für die gesunden Teilnehmer schwierig nachvollziehbar.

Wir haben keine Fragen hinzugefügt bzw. entfernt und keine Änderungen in den Antwortkategorien vorgenommen.

Die endgültige deutsche Version der BBPDS-D findet sich in Anhang 2. 


\section{Validitätsprüfung}

Die Ergebnisse der Valditätsprüfung sind in Tabelle 2 zusammengefasst. Die relative Zuverlässigkeit, welche mittels ICC $_{2,1}$ ermittelt wurde, erwies sich in allen drei Gruppen als ausgezeichnet (gesamte Studienpopulation, Patienten mit akutem bzw. stabilem CRPS).

Die Crohnbach Alpha-Werte ergaben in allen drei Gruppen eine exzellente interne Konsistenz und Zuverlässigkeit. Die SDC lag insgesamt bei 10,0 Punkten (akutes CRPS 10,7, stabiles CRPS 9,9). Wie im Bland-Altman Plot ersichtlich, lagen für den Test-Retest 48 Punkte innerhalb des 95-Prozent-Konfidenzintervalls (Abbildung 1). Die Sichtprüfung ergab keine Tendenz zur Heteroskedastizität. Die Spearmans- $\rho$-Werte zeigten keine Korrelation zwischen dem BBPDS-D Gesamtwert, den Werten der NRS $(\rho=-0,19)$ und dem EuroQol-5D ( $\rho=$ 0,16). Die Mann-Whitney-U-Tests zeigten keine signifikanten Unterschiede zwischen Patienten mit akutem und stabilem CRPS $(\mathrm{p}=0,412)$. Es gab keinerlei Boden- oder Deckeneffekte. 


\section{Diskussion}

Die Ergebnisse der vorliegenden Studie zeigen, dass die deutsche Übersetzung der englischen Originalversion des BPDS unter Berücksichtigung kultureller und linguistischer Anpassungen ein valides und konzises Werkzeug zur Quantifizierung von BPD bei deutschsprachigen Patienten mit akutem bzw. stabilem CRPS Typ darstellt.

Kliniker und Wissenschaftler auf dem Gebiet des CRPS haben nun die Möglichkeit Körperwahrnehmungsstörungen bei deutschsprachigen CRPS Patienten zu erfassen und zu quantifizieren. Zudem kann der Fortschritt des Behandlungsverlaufes individuell monitorisiert werden.

Die erreichte Punktzahl unserer Studienteilnehmer steht in Einklang zu den Resultaten einer früheren Untersuchung von Lewis et al. [9], was die Konsistenz des vorliegenden BPDS-D unterstreicht. Obwohl Körperwahrnehmungsstörungen beim CRPS häufig auftreten, hat die Wissenschaft bisher wenig Aufmerksamkeit auf dieses Phänomen gerichtet. Im Jahr 2012 untersuchte Lewis et al. die Beziehung zwischen Körperwahrnehmungsstörungen, Schmerzen und taktiler Diskriminierung bei CRPS Patienten und gesunden Kontrollen [9]. Die Resultate zeigten, dass CRPS Patienten mit ausgeprägteren Körperwahrnehmungsstörungen an stärkeren Schmerzen litten und eine schlechtere taktile Diskriminierung in der betroffenen Extremität aufwiesen.

Es kann vermutet werden, dass das Ausmass der Körperwahrnehmungsstörungen den Therapieerfolg und der Heilungsprognose bei CRPS Patienten massgeblich beeinflusst. Daher sollte die zukünftige Forschung die zugrundeliegenden pathophysiologischen Prozesse der Körperwahrnehmungsstörungen weiter untersuchen, damit deren Auswirkungen auf das Rehabilitationsergebnis und die Prognose individuell beurteilt werden können. 
Um die Beziehung zwischen Körperwahrnehmungsstörungen, klinischer Manifestation und deren Auswirkungen auf die Aktivitäten und Partizipation zu untersuchen, sind weitere Studien nötig.

Bemerkenswerterweise offenbarten die Ergebnisse unserer Studie keine Korrelation zwischen der erreichten Punktzahl auf der BBPDS-D, dem Schmerz bzw. der Lebensqualität. Eine mögliche Hypothese wäre, dass das die Körperwahrnehmungsstörungen selbst nicht durch NSR und EQ-5D nicht entsprechend objektiviert werden kann, was die Wichtigkeit des Einsatzes der BBPDS im klinischen Alltag und in Studien unterstreicht.

Eine Stärke der Studie beinhaltet die Verwendung einer robusten Methodik gemäss etablierten Richtlinien der amerikanischen Vereinigung der Orthopädischen Chirurgen (AAOS) [12]. So haben wir dafür gesorgt, dass der Inhalt, die Integrität und die Essenz der Fragen der Orginalversion in der Zielsprache Deutsch beibehalten werden. Mit dem Einschluss von Patienten mit akutem und stabilem CRPS wurde zudem der Heterogenität der Erkrankung Rechnung getragen, da die klinischen Manifestationen sich im Verlauf der Erkrankung charakteristischerweise ändern [19].

Eine Einschränkung der vorliegenden Studie stellt die relativ kleine Stichprobe dar. Diese hat potenziell einen Einfluss auf die psychometrischen Eigenschaften. Eine weitere Einschränkung betrifft das Fehlen einer konvergenten Validierung der BBPDS-D. Der Neglekt Score - eingeführt durch Galer und Jensen - ist zwar ein vergleichbares Instrument, wurde nach unseren Kenntnissen jedoch bisher nicht in englischer Sprache validiert [5]. 


\section{Fazit für die Praxis}

Die vorliegende deutsche Übersetzung und interkulturelle Anpassung der BBPDS stellt ein valides Instrument dar, um Körperwahrnehmungsstörungen bei deutschsprachigen CRPS Patienten mit BPD zu objektivieren und zu quantifizieren. In zukünftigen Forschungsprojekten sollte der Einfluss von Körperwahrnehmungsstörungen auf das Behandlungsergebnis und die Prognose des CRPS weiter untersucht werden. 


\section{Referenzen}

1. Stanton-Hicks M, Janig W, Hassenbusch S, Haddox JD, Boas R, Wilson P: Reflex sympathetic dystrophy: changing concepts and taxonomy. Pain 1995, 63(1):127-133.

2. Marinus J, Moseley GL, Birklein F, Baron R, Maihofner C, Kingery WS, van Hilten JJ: Clinical features and pathophysiology of complex regional pain syndrome. Lancet Neurol 2011, 10(7):637-648.

3. Harden R, Bruehl S: CRPS: Current Diagnosis and Therapy. In: Diagnostic criteria: The statistical derivation of the four criterion factors edn. Edited by Wilson P, Stanton-Hicks M, Harden R. Seattle, WA: IASP Press; 2005: 45-58.

4. Forderreuther S, Sailer U, Straube A: Impaired self-perception of the hand in complex regional pain syndrome (CRPS). Pain 2004, 110(3):756-761.

5. Galer BS, Jensen M: Neglect-like symptoms in complex regional pain syndrome: results of a self-administered survey. J Pain Symptom Manage 1999, 18(3):213-217.

6. Lewis JS, Kersten P, McPherson KM, Taylor GJ, Harris N, McCabe CS, Blake DR: Wherever is my arm? Impaired upper limb position accuracy in complex regional pain syndrome. Pain 2010, 149(3):463-469.

7. Lewis JS, Kersten P, McCabe CS, McPherson KM, Blake DR: Body perception disturbance: a contribution to pain in complex regional pain syndrome (CRPS). Pain 2007, 133(1-3):111-119.

8. Galer BS, Butler S, Jensen MP: Case reports and hypothesis: a neglect-like syndrome may be responsible for the motor disturbance in reflex sympathetic dystrophy (Complex Regional Pain Syndrome-1). J Pain Symptom Manage 1995, 10(5):385-391. 
9. Lewis JS, Schweinhardt P: Perceptions of the painful body: the relationship between body perception disturbance, pain and tactile discrimination in complex regional pain syndrome. Eur J Pain 2012, 16(9):1320-1330.

10. Lewis JS, McCabe C: Body perception disturbance (BPD) in CRPS. Pract Pain Manag 2010(10):60-66.

11. Harden RN, Bruehl S, Perez RS, Birklein F, Marinus J, Maihofner C, Lubenow T, Buvanendran A, Mackey S, Graciosa J et al: Validation of proposed diagnostic criteria (the "Budapest Criteria") for Complex Regional Pain Syndrome. Pain 2010.

12. Beaton DE, Bombardier C, Guillemin F, Ferraz MB: Guidelines for the process of cross-cultural adaptation of self-report measures. Spine (Phila Pa 1976) 2000, 25(24):3186-3191.

13. von der Schulenburg JM, Claes C, Greiner W: Die deutsche Version des EuroQolFragebogens. Z Gesundheitswissenschaften 1998, 6:3-20.

14. Swanenburg J, Humphreys K, Langenfeld A, Brunner F, Wirth B: Validity and reliability of a German version of the Neck Disability Index (NDI-G). Man Ther 2014, 19(1):52-58.

15. Cicchetti DC: Guidelines, criteria, and rules of thumb for evaluating normed and standardized assessment instruments in psychology. Psychological Assessment 1994, 6(4):284-290.

16. de Vet HCW, Terwee CB, Knol DL, Bouter LM: When to use agreement versus reliability measures. Journal of Clinical Epidemiology 2006, 59(10):1033-1039.

17. Bland JM, Altman DG: Statistical methods for assessing agreement between two methods of clinical measurement. Lancet 1986, 1(8476):307-310. 
18. Siegel S, Castellan JNJ: Non parametric statistics for the behavioral science, 2nd edn: McGraw-Hill Inc.; 1988.

19. Veldman PH, Reynen HM, Arntz IE, Goris RJ: Signs and symptoms of reflex sympathetic dystrophy: prospective study of 829 patients. Lancet 1993, 342(8878):1012-1016. 
Tabelle 1: Demographische und klinische Daten $(\mathrm{N}=56)$

\begin{tabular}{|c|c|c|c|}
\hline & $\begin{array}{l}\text { Akutes CRPS } 1 \\
(\mathrm{~N}=\mathbf{2 8})\end{array}$ & $\begin{array}{l}\text { Stabiles CRPS } 1 \\
(\mathrm{~N}=28)\end{array}$ & $\rho$-Wert \\
\hline Frauen & 25 & 24 & n.a. \\
\hline Männer & 3 & 4 & n.a. \\
\hline Alter; Jahre (MW, SD) & $50.1(15.3)$ & $51.7(10.7)$ & 0.97 \\
\hline Altersspanne; Jahre (MW, SD) & $18-77$ & $26-71$ & n.a. \\
\hline Gewicht; kg (MW, SD) & $68.6(15.3)$ & $72.5(17.3)$ & 0.500 \\
\hline Grösse; cm (MW, SD) & $165.8(5.0)$ & $167.9(10.7)$ & 0.005 \\
\hline Symptomdauer; Monate (MW, & $3.1(0-3,2.6)$ & $41(3-240,52.2)$ & $<0.001$ \\
\hline \multicolumn{4}{|l|}{ Spanne, SD) } \\
\hline \multicolumn{4}{|l|}{ Betroffene Seite } \\
\hline Rechts & 16 & 16 & n.a. \\
\hline Links & 12 & 12 & n.a. \\
\hline \multicolumn{4}{|l|}{ Lokalisation } \\
\hline Obere Extremität & 18 & 11 & n.a. \\
\hline Untere Extremität & 10 & 17 & n.a. \\
\hline \multicolumn{4}{|l|}{ BBPDS-D (MW, SD) } \\
\hline 1. Messung & $19.8(9.6)$ & $22.0(9.3)$ & 0.324 \\
\hline 2. Messung & $18.4(9.0)$ & $20.7(9.6)$ & 0.756 \\
\hline EQ-5D (MW, SD) & $0.64(1.9)$ & $0.65(0.17)$ & 0.360 \\
\hline NRS (MW, SD) & $54.3(21.8)$ & $57.2(19.5)$ & 0.594 \\
\hline
\end{tabular}


NRS: Numeric rating scale $(0=$ kein Schmerz, $100=$ maximaler Schmerz $)$, MW: Mittelwert, SD: Standarddeviation; BBPDS-D: Deutsche Version der Bath Body Perception Disturbance Scale 
Tabelle 2: Test-Retest-Zuverlässigkeit und Resultate der BBPDS-D (N=56)

\begin{tabular}{|c|c|c|c|c|c|}
\hline BBPDS-D & 1. Messung & 2. Messung & $\begin{array}{l}I^{I C C_{2,1}} \\
(95 \% \mathrm{CI})\end{array}$ & $\begin{array}{l}\text { Crohnbac } \\
\text { hs Alpha } \\
\text { (1. } \\
\text { Messung) }\end{array}$ & SDC \\
\hline Total (MW, SD) & $20.9(9.4)$ & $19.5(9.3)$ & $0.85(0.75,0.91)$ & 0.92 & 10.0 \\
\hline Akutes CRPS (MW, SD) & $19.8(9.6)$ & $18.4(9.0)$ & $0.82(0.65,0.91)$ & 0.90 & 10.7 \\
\hline Stabiles CRPS (MW, SD) & $22.0(9.3)$ & $20.7(9.6)$ & $0.86(0.74,0.94)$ & 0.93 & 9.9 \\
\hline
\end{tabular}

BBPDS-D: Deutsche Version der Bath Body Perception Disturbance Scale, MW: Mittelwert, SD: Standarddeviation, CI: Konfidenzintervall, SDC: Minimale messbare Veränderung (smallest detectable change) 
Abblidung 1: Korrespondierender Autor 


\section{Abbildung 2: Mittelwert jedes Patienten gegenüber der Differenz zwischen dem Test-Retest-Resultat der BBPDS-D}

BBPDS-D: Deutsche Version der Bath Body Perception Disturbance Scale, SD: Standard deviation 